\title{
The Future of Paediatric Surgery in Nepal
}

\author{
Thapa NB ${ }^{1}$ \\ ${ }^{1}$ Dr. N.B. Thapa Consultant Paediatric Surgeon, Ishan Children's Hospital, Kathmandu, Nepal.
}

Address for Correspondence: Dr. N.B. Thapa, E-mail: narayan.thapa@gmail.com

In 1981, I returned as a paediatric surgeon to Nepal. Dr. Keshab B. Rajbhandary followed me a little later. The two of us had the monumental task of establishing a new surgical speciality in the country. Kanti Children's Hospital $(\mathrm{KCH})$ was not yet developed as a surgical centre. The first thing we did was to join the newly formed Nepal Paediatric Society as full members. It was a good sign that our medical colleagues welcomed us in their midst. We could count on their cooperation. But progress was slow because of the lack of basic infrastructure. Starkly stated, there was no functioning operation theatre (water leakage), no paediatric surgical instruments and equipment (only the gross sizes of Russian instruments) and crucially, no paediatric anaesthetist on the staff. There were no neonatal units. Conditions were not much better for medical specialities. I wrote an article about our subject in an English language daily and we put our heads down to chase the authorities. In those initial years, we had some important achievements:

1. I was able to organize a rotation of British anaesthetists on local pay for five years to supplement the Nepalese anaesthetists, who in turn became increasingly adept at paediatric anaesthesia.

2. A first phase of Japanese aid totalling US\$2 million was negotiated for $\mathrm{KCH}$ to buy equipment. This was the first step towards modernity in medical and surgical paediatric practice in Nepal. Credit for this goes to the late Mr. Ryutaro Hashimoto, who was a member of the Diet at the time, and was later to become the Prime Minister of Japan. He took a personal interest and visited $\mathrm{KCH}$ many times. Incubators, ventilators, monitors and anaesthetic machines became available and all forms of paediatric surgery were begun, including Neonatal Surgery.

3. This was followed by Japanese funding on a much larger scale, accorded to $\mathrm{KCH}$ for the building expansion culminating in the present set-up, with modern Operating Theatres and a Neonatal Surgical Intensive Care Unit. Ancillary services such as a modern Radiology Unit were added along with similar improvements in the medical side of paediatrics.

This is how things stood about 15 years ago. The number of paediatric surgeons now stands at eight in the country. The number of operations performed has increased substantially. However, the range of operations has remained limited to that of those early days.

Six areas need to be looked at in order to improve the surgical service delivered to our children.

\section{Equipment}

There is a need to upgrade the equipment at $\mathrm{KCH}$. As it is a tertiary referral centre and a teaching centre, this is an absolute must. There are huge deficiencies in the armamentarium available to paediatric surgeons. How difficult it has been, for example, to deal with patients who suffer from Hirschprung's Disease without the facility for frozen section. This is long overdue and a shameful oversight. In some western children's hospitals more than 50 percent of surgery is done laparoscopically these days. However, we have not even made a start in this field. Although basic endoscopic facilities were part of the original Japanese package, it remains basic and mostly unused. Ditto for the urologic set-up, this is basic and quite old. No one has even thought about a CT scan or a MRI unit for $\mathrm{KCH}$. This stagnancy is a great obstacle to progress.

\section{Knowledge Update}

As is true for many other specialties in Nepal, relevant journals are not available. Free internet access to journals is very limited and paying for it is truly expensive. Libraries lack reference literature. There is limited discussion among professionals. All this points to an unwillingness to put too much effort into this area. Needless to say change is urgently needed.

One other area of concern for all medical professionals is the lack of financial wherewithal to attend international conferences. In a country where millions of dollars are spent on treatment abroad for politicians, presumably for more expertise than available here, it is a wonder that the medical establishment has never insisted that the government spend some money on facilitating the acquisition of expertise for our own specialists. Medical professionals would be able to keep in touch with recent developments by attending international conferences in their own field. Then perhaps the ordinary people of this country would also be able to avail themselves of high expertise locally.

\section{Regional Centres}

There is, as yet, no move to regionalize paediatric surgical services. Under the present circumstances this might take years. This is a pity because having to travel long distances to Kathmandu may not be economically feasible for many. During emergencies this may simply not be possible.

\section{Information}

What the general public knows about operations on children is naturally very limited simply because they have 
not been told about it. Many general physicians tell the parents that surgery should be deferred until the child grows up. This does great disservice to the child because many operable conditions may be causing pain or discomfort and may lead to emergencies. At the very least, there may be psychological distress that needs to be considered. A simple referral is the best course.

\section{Training}

At $\mathrm{KCH}$, the waiting period for routine surgery is in the region of two years. Most of the present numbers of paediatric surgeons (eight) undertook to obtain training on their own initiative. It is high time that along with the setting up of regional centres for this speciality, more government sponsored training for additional surgeons should be initiated. Of course, one should not forget that specialized training is needed for other important professionals such as nurses and anaesthetists, and the parallel development of paediatric medicine should be a given.

\section{Development of Subspecialties}

As future development, one can look forward to establishment of units in plastic surgery, orthopaedic surgery, urologic and cardio-thoracic surgery. Acquisition of trained manpower and equipment has to go hand in hand.

What are mentioned above are the essentials under the present financial situation the country is passing through. In due course, one would hope the health services would be able to afford countrywide neonatal ambulances service for transferring of neonates for tertiary care - these ambulances will have incubators and other facilities for safe transfers. Data on congenital abnormalities needs to be collected and public need to be educated. Paediatric surgeons themselves already can be doing something about it. Same goes for research in the field.

Lastly, one need not look anywhere else for the paediatric surgeons to start forming a group to discuss common problems and exchange of experiences and information. 